\title{
Trauma Hemostasis and Oxygenation Research Network position paper on the role of hypotensive resuscitation as part of remote damage control resuscitation
}

Woolley, Thomas, MD; Thompson, Patrick; Kirkman, Emrys, PhD; Reed, Richard; Ausset, Sylvain, MD; Beckett, Andrew, MD; Bjerkvig, Christopher, MD; Cap, Andrew, P., MD, PhD; Coats, Tim, MD; Cohen, Mitchell, MD; Despasquale, Marc; Dorlac, Warren, MD; Doughty, Heidi; Dutton, Richard, MD; Eastridge, Brian; Glassberg, Elon, MD; Hudson, Anthony; Jenkins, Donald, MD; Keenan, Sean, MD; Martinaud, Christophe, PhD; Miles, Ethan; Moore, Ernest, MD; Nordmann, Giles; Prat, Nicolas, PhD; Rappold, Joseph, MD; Reade, Michael, C., MBBD D Phil; Rees, Paul, MD; Rickard, Rory, PhD; Schreiber, Martin, MD; Shackelford, Stacy, MD; Skogran Eliassen, Håkon; Smith, Jason, MD; Smith, Mike, PhD; Spinella, Philip, MD; Strandenes, Geir, MD; Ward, Kevin, MD; Watts, Sarah, PhD; White, Nathan, MD; Williams, Steve

Journal of Trauma and Acute Care Surgery: June 2018 - Volume 84 - Issue 6S - p S3-S13 doi: 10.1097/TA.0000000000001856

Thor 2018

\begin{abstract}
Author Information
ABSTRACT The Trauma Hemostasis and Oxygenation Research (THOR) Network has developed a consensus statement on the role of permissive hypotension in remote damage control resuscitation (RDCR). A summary of the evidence on permissive hypotension follows the THOR Network position on the topic. In RDCR, the burden of time in the care of the patients suffering from noncompressible hemorrhage affects outcomes. Despite the lack of published evidence, and based on clinical experience and expertise, it is the THOR Network's opinion that the increase in prehospital time leads to an increased burden of shock, which poses a greater risk to the patient than the risk of rebleeding due to slightly increased blood pressure, especially when blood products are available as part of prehospital resuscitation.
\end{abstract}

The THOR Network's consensus statement is, "In a casualty with life-threatening hemorrhage, shock should be reversed as soon as possible using a blood-based HR fluid. Whole blood is preferred to blood components. As a part of this HR, the initial systolic blood pressure target should be $100 \mathrm{~mm} \mathrm{Hg}$. In RDCR, it is vital for higher echelon care providers to receive a casualty with sufficient physiologic reserve to survive definitive surgical hemostasis and aggressive resuscitation. The combined use of blood-based resuscitation and limiting systolic blood pressure is believed to be effective in promoting hemostasis and reversing shock"

From the Academic Department of Military Anaesthetics and Critical Care (T.W.), Royal Centre for Defence Medicine, Birmingham, United Kingdom; UK Paramedic (P.T.), THOR Steering Committee, Scotland; Combat Casualty Care (E.K.), Medical \& Trauma Sciences, Defence Science and Technology Laboratory, Wiltshire; 16 Medical Regiment (R.R.), Colchester, United Kingdom; Anaesthesia and Intensive Care (S.A.), Val de Grâce Military Academy, Paris, France; Royal Canadian Medical Services (A.B.), McGill University, Montreal, Canada; Department of Anaesthesia and Intensive Care (C.B.), Haukeland University Hospital, Bergen, Norway; Coagulation and Blood Research (A.C.), US Army Institute of Surgical Research, San Antonio, Texas; Emergency Medicine (T.C.), University of Leicester, Leicester, United Kingdom; Surgery (M.C.), University of Colorado School of Medicine, Aurora; Paramedic NREMT (M.D.); Trauma/Acute Care Surgery (W.D.), University of Colorado Health, Denver, Colorado; Transfusion Medicine (H.D.), NHS Blood and Transplant, Watford, United 
Kingdom; Chief Quality Officer (R.D.); Department of Surgery (B.E.), UT Health Science Center, San Antonio, Texas; Israel Defense Forces Medical Corps. (E.G.), Bar-Ilan University Faculty of Medicine, Safed, Israel; Peninsula Trauma Network (A.H.), Royal Devon \& Exeter NHS Foundation Trust, Exeter, United Kingdom; Division of Trauma and Emergency Surgery (D.J.), UT Health San Antonio, San Antonio, Texas; Special Operations Command Europe (S.K.), Stuttgart, Germany; Transfusion Medicine and Cell Therapy (C.M.), French Military Blood Institute, Clamart, France; 75th Ranger Regiment (E.M.); Surgery (E.M.), University of Colorado Denver, Denver, Colorado; Military Anaesthesia (G.N.), Royal Centre for Defence Medicine, Birmingham, United Kingdom; Combat Casualty Care (N.P.), French Armed Forces Institute of Biomedical Research; Acute Care Surgery, Toulon, France (J.R.), Tufts University School of Medicine, Boston, Massachusetts; Military Medicine and Surgery (M.R.), Australian Defence Force Joint Health Command and Faculty of Medicine, University of Queensland, Queensland, Australia; Interventional Cardiology, Acute \& Prehospital Medicine (P.R.), University of St Andrews School of Medicine, St Andrews, Scotland; Department of Military Surgery and Trauma (R.R.), Royal Centre for Defence Medicine, Birmingham, United Kingdom; Division of Trauma, Critical Care \& Acute Care Surgery (M.S.), Oregon Health Sciences University, Portland, Oregon; Department of Defense (S.S.), Joint Trauma System; Norwegian Naval Special Operations Commando (H.S.), University of Bergen, Bergen, Norway; Department of Emergency Medicine (J.S.), General Practise (M.S.), Royal Centre for Defence Medicine, Birmingham, United Kingdom; Department of Pediatrics (P.S.), Washington University in St Louis School of Medicine, St Louis, Missouri; Department of Immunology and Transfusion Medicine (G.S.), Norwegian Armed Forces Medical Services, Oslo, Norway; Department of Emergency Medicine (K.W.), University of Michigan, Ann Arbor, Michigan; Combat Casualty Care (S.W.), Medical \& Trauma Sciences, Defence Science and Technology Laboratory, Salisbury, United Kingdom; Division of Emergency Medicine (N.W.), Washington University of Medicine, Seattle, Washington; and Institute of Cruise Ship Medicine (S.W.), REVA Air Ambulance, Fort Lauderdale, Florida.

Submitted: December 10, 2017, Revised: February 6, 2018, Accepted: February 7, 2018, Published online: February 27, 2018.

Paper presented at the THOR conference 2017.

Address for reprints: Thomas Woolley, MD, Royal Centre for Defence Medicine, ICT Centre, Birmingham Research Park, Vincent Drive, Birmingham, B15 2SQ, United Kingdom; email: tomwoolley@mac.com.

(C) 2018 Lippincott Williams \& Wilkins, Inc.

This website uses cookies. By continuing to use this website you are giving consent to cookies being used. For information on cookies and how you can disable them visit our Privacy and Cookie Policy.

Got it, thanks! 